Supporting Information

\title{
Lithium Transport Pathways Guided by Grain Architectures in Ni-Rich Layered Cathodes
}

\author{
Yuki Nomura $^{\dagger^{*}}$, Kazuo Yamamoto ${ }^{*}$, Yuji Yamagishi ${ }^{\dagger}$, Emiko Igaki $^{\dagger}$ \\ 'Technology Division, Panasonic Corporation, 3-1-1 Yagumo-naka-machi, Moriguchi, Osaka 570-8501, \\ Japan. \\ ${ }^{7}$ Nanostructures Research Laboratory, Japan Fine Ceramics Center, 2-4-1 Mutsuno, Atsuta-ku, Nagoya, Aichi \\ 456-8587, Japan.
}

Table of contents

Electrochemical characteristics of the solid-state Li-ion battery

Influence of electron irradiation

Tensor decomposition

Quantification of Li concentration

Automated crystal orientation mapping

Twin in NCA

References 


\section{Electrochemical characteristics of the solid-state Li-ion battery}

The charge and discharge curves and electrochemical impedance spectra are shown in Fig. S1. The charge/discharge measurements shown in Fig. S1(a) were performed at a constant current of $2.36 \mu \mathrm{A}$ using a potentio/galvanostat (SP-300, Bio-logic Science Instruments). The cut-off potentials for charging and discharging were set to 3.7 and $1.9 \mathrm{~V}$, respectively. The electrochemical impedance spectroscopy results shown in Fig. S1(c)-(f) were performed at a frequency range of $7 \mathrm{MHz}$ to $100 \mathrm{mHz}$ using a potentio/galvanostat. Note that the sizes of battery are different for the sample in Fig. S1 (pellet in an area of $70.8 \mathrm{~mm}^{2}$ ) and the one in Fig. $1(\mathrm{c})$ (pellet in an area of less than $1 \mathrm{~mm}^{2}$ ).

(a)

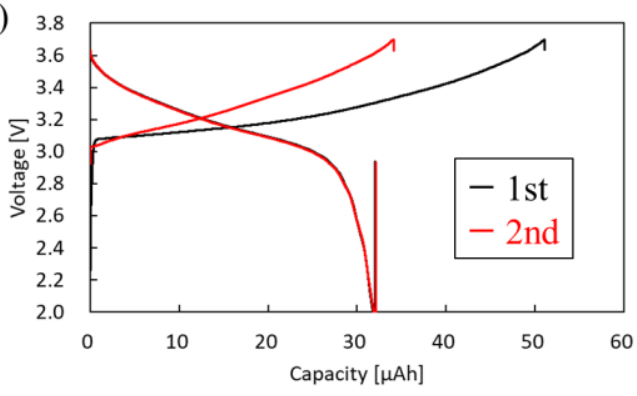

(b)

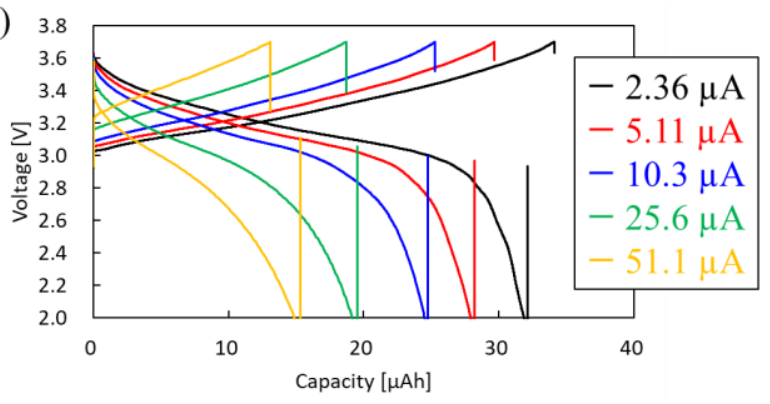

(c)

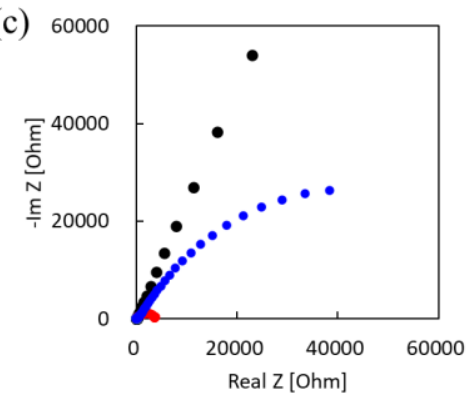

(d)

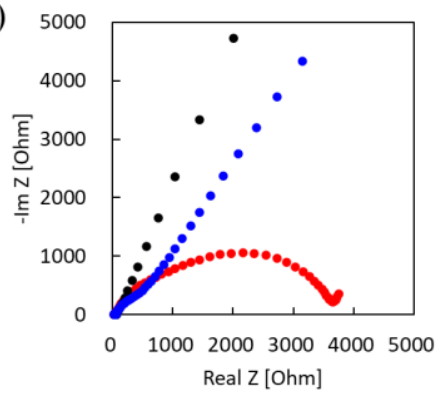

(e)

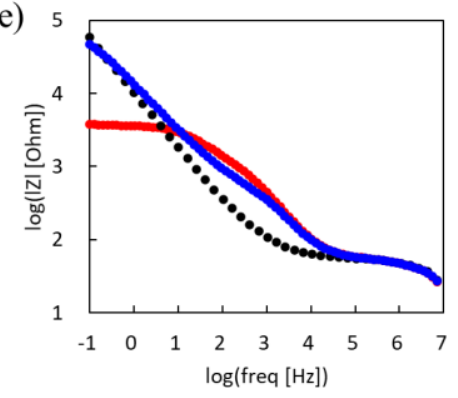

(f)

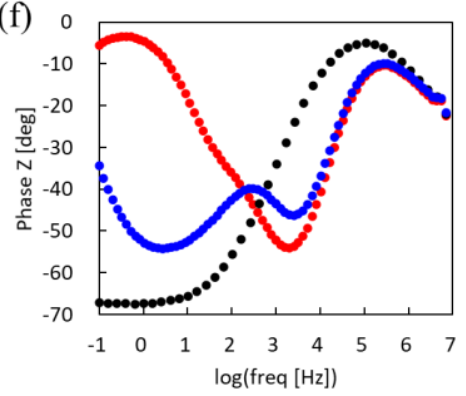

pristine charged discharged

Figure S1. Electrochemical behavior of the solid-state Li-ion battery. (a) Charge/discharge curves for first two cycles at $2.36 \mu \mathrm{A}$. (b) Rate characteristics. (c), (d) Cole-Cole plots. (e), (f) Bode plots. 


\section{Influence of electron irradiation}

The effect of electron irradiation is discussed in the Supplementary Information of our previous study. ${ }^{1}$ The total electron dose in this experiment was approximately $1.0 \times 10^{5}$ electron $/ \mathrm{nm}^{2}$, which is two orders of magnitude lower than that of atomic-resolution STEM $\left(\sim 2.8 \times 10^{7}\right.$ electron $\left./ \mathrm{nm}^{2}\right){ }^{2}$ Thus, we believe that a deterioration of the NCA crystal structure did not occur in our experiment.

\section{Tensor decomposition ${ }^{3,4}$}

Figure S2(a) shows a fourth-order tensor, $\boldsymbol{X} \in \mathbb{R}^{I \times J \times K \times L}$, showing a 4D time-resolved STEMEEL spectral image, where $I, J, K$, and $L$ are the lengths of spatial-x, spatial-y, energy-loss, and time, respectively. Tensor decomposition learns the $n$-dimensional features of the signals. However, spatial-x and spatial-y do not have any principal components. Thus, matrices on the spatial-x and spatial-y planes are converted to vectors and are arranged into the rows of a new third-order tensor, $\mathcal{X}^{\prime} \in \mathbb{R}^{I J \times K \times L}$, (Fig. S2(b)). The third-order tensor $\boldsymbol{X}^{\prime}$ is decomposed into a core tensor, $\mathcal{G} \in \mathbb{R}^{P \times Q \times R}(I J \geq P, K \geq Q, L \geq R)$, and three factor matrices, $\boldsymbol{U} \in \mathbb{R}^{I J \times P}, \boldsymbol{V} \in$ $\mathbb{R}^{K \times Q}$, and $\boldsymbol{W} \in \mathbb{R}^{L \times R}$, using Tucker decomposition, as shown in Fig. S2(c). The three factor matrices contain the principal components of each dimension. Then, a denoised third-order tensor $\boldsymbol{X}^{\prime \prime} \in \mathbb{R}^{I J \times K \times L}$ was reconstructed using $\mathcal{G}, \boldsymbol{U}, \boldsymbol{V}$, and $\boldsymbol{W}$. If $P, Q$, and $R$ are smaller than $I J$, $K$, and L, the tensor $\mathcal{X}^{\prime \prime} \in \mathbb{R}^{I \times K \times L}$ is the denoised version of the original tensor $\boldsymbol{X}^{\prime}$ (Fig. S2(d)). Finally, rows in the denoised tensor $\mathcal{X}^{\prime \prime}$ are converted to matrices on the spatial-x and spatial-y plane and combined to form a denoised fourth-order tensor $\mathcal{X}^{\prime \prime \prime} \in \mathbb{R}^{I \times J \times K \times L}$, showing a denoised 4D time-resolved STEM-EEL spectral image. In the present study, P, Q, and R were set to 33, 5, and 10 , respectively. 


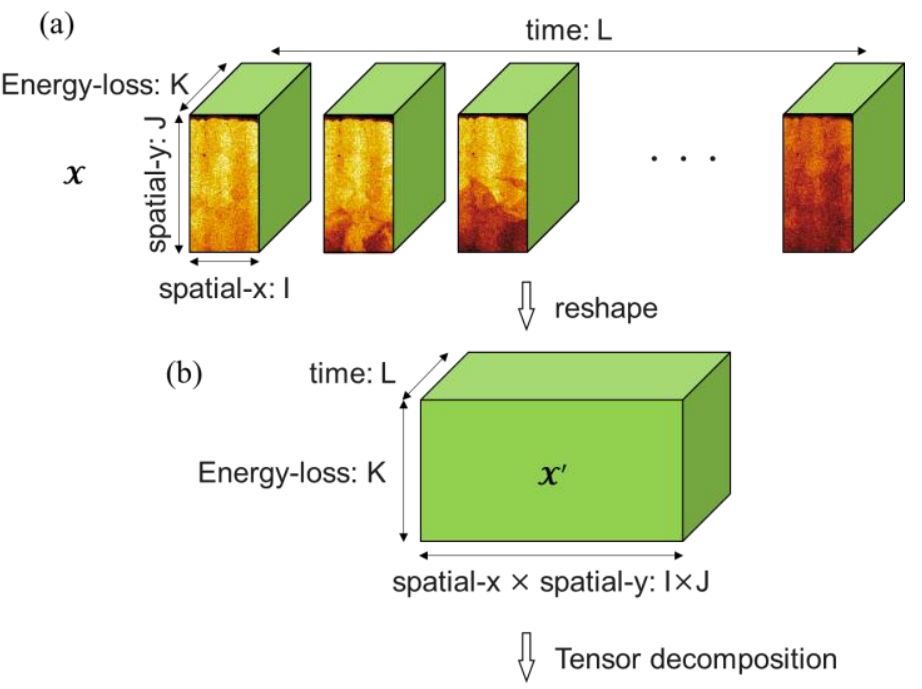

(c)

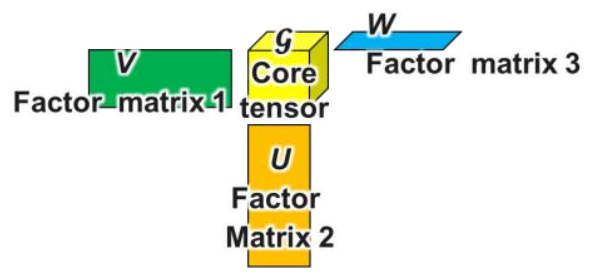

(d)
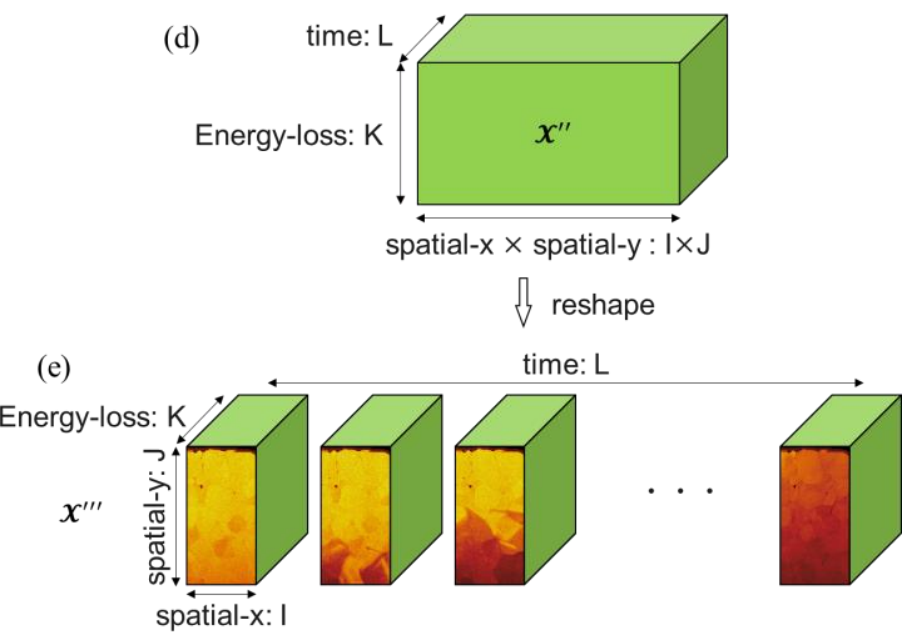

Figure S2. Tensor decomposition for time-resolved STEM-EELS. (a) 4D data showing a timeresolved STEM-EEL spectral image. (b) Reshaped 3D data. Spatial matrices are converted to vectors. (c) Tucker decomposition of the third-order tensor. The third-order tensor is decomposed into a core tensor, $\boldsymbol{G}$, and three factor matrices, $\boldsymbol{U}, \boldsymbol{V}$, and $\boldsymbol{W}$. The factor matrices statistically represent the principal components of each dimension. The core tensor indicates the levels of interaction between the different components for each dimension. (d) Denoised 3D data. (e) Denoised 4D data showing a denoised time-resolved STEM-EEL spectral image. 


\section{Quantification of $\mathrm{Li}$ concentration}

Figure S3(a) shows an ADF-STEM image and a thickness map (inelastic mean free path, $\lambda$ ) of the scanned area for in situ STEM-EELS. The results show that the lower region is thicker than the upper region. Figure S3(b) shows the ADF-STEM image and the denoised Li-K edge intensity maps. The core-loss signal generally decreases with an increase in the sample thickness owing to multiple scatterings of the incident electrons. Thus, the Li-K edge intensity in the lower area is slightly lower than that in the upper area, as shown in the b2 panel of Fig. S3(b), despite the initial $\mathrm{Li}$ concentration in the entire area was constant $\left(\mathrm{x}=1.0\right.$ in $\left.\mathrm{Li}_{\mathrm{x}} \mathrm{Ni}_{0.8} \mathrm{Co}_{0.15} \mathrm{Al}_{0.05} \mathrm{O}_{2}\right)$. To remove the thickness effect, the b3-b12 panels were divided by the b2 panel. The results are shown in $\mathrm{c} 3-\mathrm{c} 11$. Moreover, average $\mathrm{Li}$ concentration before charging and after $3.7 \mathrm{~V} v s . \mathrm{In} / \mathrm{Li}$ charging (c12 panel) is known to be approximately $x=1.0$ and $0.2 .{ }^{5}$ Using this value, we converted the standardized $\mathrm{Li}-\mathrm{K}$ intensities to quantitative $\mathrm{Li}$ concentrations. Note that the intensity is a linear combination with the $\mathrm{Li}$ concentration $\left(\mathrm{x}\right.$ in $\left.\mathrm{Li}_{\mathrm{x}} \mathrm{Ni}_{0.8} \mathrm{Co}_{0.15} \mathrm{Al}_{0.05} \mathrm{O}_{2}\right){ }^{6}$ as shown in the main text.

(a)

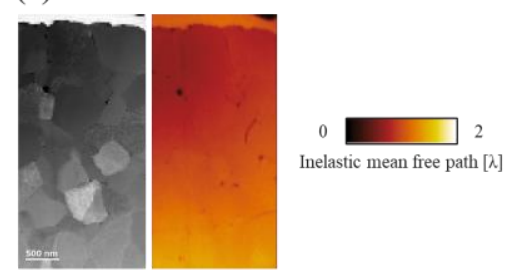

(b)
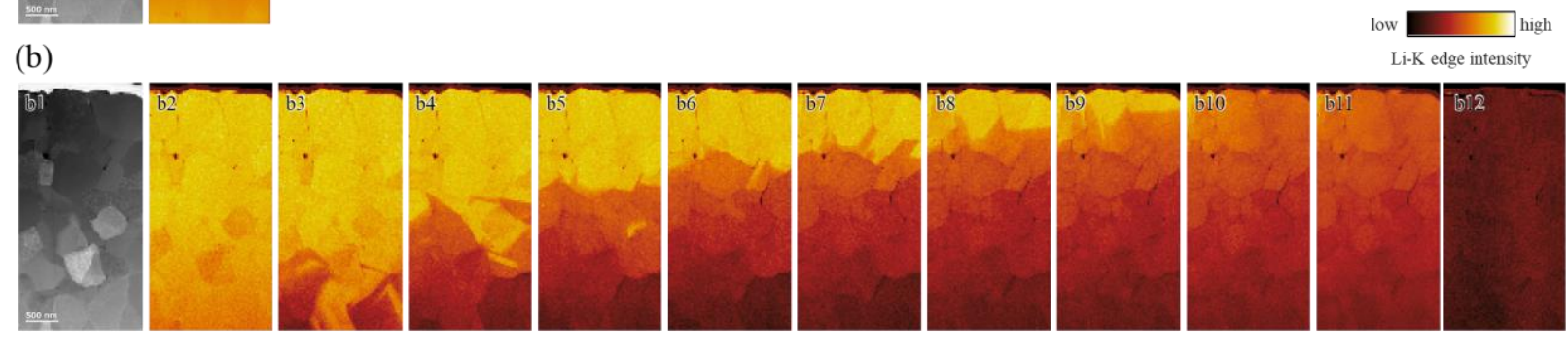

(c)
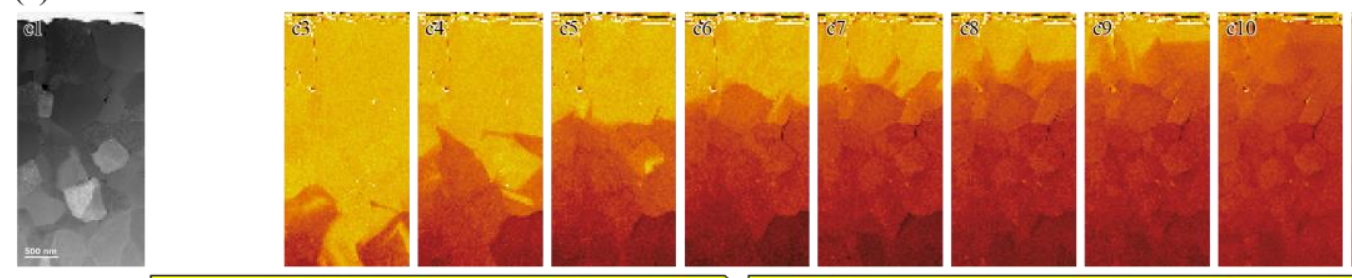

$0.0 \square$ in $\mathrm{Li}_{\mathrm{x}} \mathrm{Ni}_{0.8} \mathrm{Co}_{0.15} \mathrm{Al}_{0.05} \mathrm{O}_{2}$
1.3 Relaxation (open circuit)

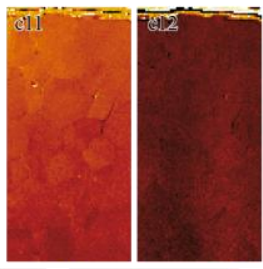

Figure S3. Quantification of Li concentrations. (a) ADF-STEM image and thickness map of the scanned area for in situ STEM-EELS. (b) Li-K intensity maps denoised by tensor decomposition. (c) Quantified Li concentration maps. 


\section{Automated crystal orientation mapping ${ }^{7,8}$}

An automated crystal orientation mapping (ACOM) is a method used for mapping the crystal orientations and phases of grains. Hardware and software for ACOM is currently commercialized by NanoMEGAS SPRL (ASTAR, DigiSTAR, and TOPSPIN). In ACOM, diffraction patterns are serially recorded at all electron probe positions of the raster-scanned region on the sample surface with precession. Precession electron diffraction (PED) averages the diffraction patters as the beam rotates around the optic axis. Therefore, a larger number of diffraction spots are observed than without precession. The PED also suppress the dynamical diffraction effect and Kikuchi lines. In this study, the precession angle was set to $0.5^{\circ}$. For each PED pattern, the crystal phase and orientation were identified by calculating the correlation between the recorded PED pattern and all pre-simulated PED patterns for possible crystal structures (phases) and orientations. An example of a PED pattern and its identification is shown in Fig. S4. The procedure was repeated for all recorded PED patterns to map the crystal orientations and phases in the raster-scanned region.

(a)

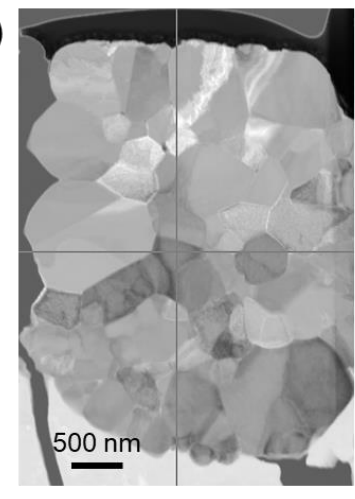

(b)

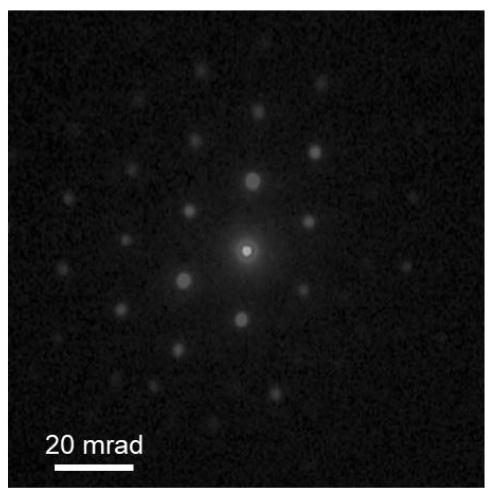

(c)

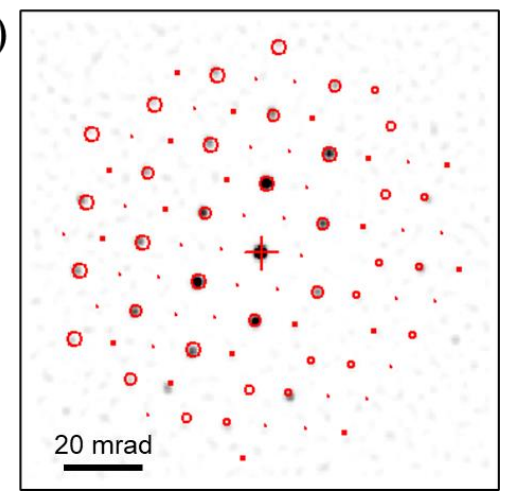

Figure S4. ACOM. (a) Bright-field STEM image. (b) PED pattern. A gray cross in (a) shows the electron probe position for (b). (c) Corresponding matched template. 


\section{Twin in NCA}

Figure S5 shows an ADF-STEM image of twins in the NCA. The structures of the twins are the same as those of $\mathrm{LiCoO}_{2}$, as shown in Fig. 4(d). ${ }^{9}$

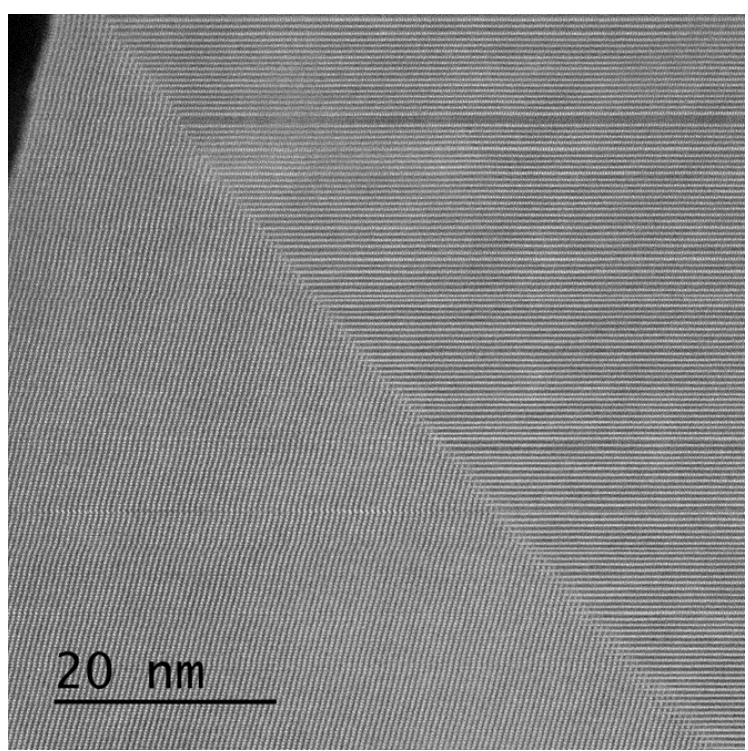

Figure S5. ADF-STEM image of the twins in the NCA.

\section{References}

(1) Nomura, Y.; Yamamoto, K.; Fujii, M.; Hirayama, T.; Igaki, E.; Saitoh, K. Dynamic Imaging of Lithium in Solid-State Batteries by Operando Electron Energy-Loss Spectroscopy with Sparse Coding. Nat. Commun. 2020, 11, 2824.

(2) Huang, R.; Ikuhara, Y. STEM Characterization for Lithium-Ion Battery Cathode Materials. Curr. Opin. Solid State Mater. Sci. 2012, 16, 31-38.

(3) Kolda, T. G.; Bader, B. W. Tensor Decompositions and Applications. SIAM Rev. 2009, 51, 455-500.

(4) Nomura, Y.; Yamamoto, K.; Anada, S.; Hirayama, T.; Igaki, E.; Saitoh, K. Denoising of Series Electron Holograms Using Tensor Decomposition. Microscopy 2021, 70, 255-264.

(5) Hwang, S.; Chang, W.; Kim, S. M.; Su, D.; Kim, D. H.; Lee, J. Y.; Chung, K. Y.; Stach, E. A. Investigation of Changes in the Surface Structure of $\mathrm{Li}_{\mathrm{x}} \mathrm{Ni}_{0.8} \mathrm{Co}_{0.15} \mathrm{Al}_{0.05} \mathrm{O}_{2}$ Cathode Materials Induced by the Initial Charge. Chem. Mater. 2014, 26, 1084-1092.

(6) Kikkawa, J.; Terada, S.; Gunji, A.; Nagai, T.; Kurashima, K.; Kimoto, K. Chemical States of Overcharged $\mathrm{LiCoO}_{2}$ Particle Surfaces and Interiors Observed Using Electron EnergyLoss Spectroscopy. J. Phys. Chem. C 2015, 119, 15823-15830.

(7) Rauch, E. F.; Portillo, J.; Nicolopoulos, S.; Bultreys, D.; Rouvimov, S.; Moeck, P. Automated Nanocrystal Orientation and Phase Mapping in the Transmission Electron Microscope on the Basis of Precession Electron Diffraction. Zeitschrift für Krist. 2010, 225, 103-109. 
(8) Brunetti, G.; Robert, D.; Bayle-Guillemaud, P.; Rouvière, J. L.; Rauch, E. F.; Martin, J. F.; Colin, J. F.; Bertin, F.; Cayron, C. Confirmation of the Domino-Cascade Model by $\mathrm{LiFePO}_{4} / \mathrm{FePO}_{4}$ Precession Electron Diffraction. Chem. Mater. 2011, 23, 4515-4524.

(9) Moriwake, H.; Kuwabara, A.; Fisher, C. A. J.; Huang, R.; Hitosugi, T.; Ikuhara, Y. H.; Oki, H.; Ikuhara, Y. First-Principles Calculations of Lithium-Ion Migration at a Coherent Grain Boundary in a Cathode Material, $\mathrm{LiCoO}_{2}$. Adv. Mater. 2013, 25, 618-622. 